\title{
AÇÕES EXTENSIONISTAS VOLTADAS PARA A PROMOÇÃO DO ENVELHECIMENTO SAUDÁVEL NO MUNICÍPIO DE CUITÉ - PB: UM RELATO DE EXPERIÊNCIA
}

\author{
Ionara de Souza JANUÁRIO ${ }^{1}$ \\ Lívia Dourado MAGALHÃES ${ }^{2}$ \\ Itacira Pedrosa de LIMA $^{3}$ \\ Anna Karla Fausto MAIA ${ }^{4}$ \\ ${ }^{1}$ Enfermeira, Universidade Federal de Campina Grande e Bolsita do Projeto de extensão "Encontro de gerações na \\ promoção do envelhecimento saudável”- Vigência - 2013 - UFCG. E-mail: ionara_76@hotmail.com \\ ${ }^{2}$ Enfermeira, Universidade Federal de Campina Grande e Voluntária do Projeto de extensão "Encontro de gerações na \\ promoção do envelhecimento saudável”- Vigência - 2013 - UFCG. E-mail: liviamagalhaes_jd@hotmail.com \\ ${ }^{3}$ Enfermeira, Universidade Federal de Campina Grande e Voluntária do Projeto de extensão "Encontro de gerações na \\ promoção do envelhecimento saudável”- Vigência - 2013 - UFCG. E-mail:tacy_pedrosa@ @otmail.com \\ ${ }^{4}$ Enfermeira. Especialista em assistência e Gestão em Saúde da Família e Auditoria. Mestranda em Gestão Hospitalar e \\ Saúde Coletiva pela Faculdade do Norte do Paraná. E-mail: akarla-maia@ hotmail.com
}

Recebido em: 30/09/2014 - Aprovado em: 30/11/2014 - Disponibilizado em: 15/12/2014

\begin{abstract}
RESUMO
OBJETIVO: relatar as ações dos extensionistas voltadas para a promoção do envelhecimento saudável no município de Cuité - PB através de um relato de experiência. METODOLOGIA: trata-se de um relato de experiência apresentando as vivências dos graduandos do curso Bacharelado em Enfermagem no desempenho de suas atividades de extensão no Projeto intitulado "Encontro de gerações na promoção do envelhecimento saudável", no período compreendido entre os meses de maio a dezembro de 2013 da Universidade Federal de Campina Grande, campus de Cuité. RESULTADOS E DISCUSSÃO: o projeto se desenvolveu a partir de seis etapas: capacitação da equipe de trabalho; aproximação com o público alvo; resgate das informações do público alvo; produção e confecção do material; educação em saúde (realização de oficinas) e produção de trabalhos científicos. Durante a vigência do projeto foram realizadas 16 oficinas e 8 temáticas diferentes foram trabalhadas. As oficinas foram realizadas com foco principal a estimulação participativa do idoso. Os temas eram explanados de forma sucinta e dinâmica, pelos extensionistas, a fim de prender a atenção dos participantes. Em seguida, os idosos interagiam de forma ativa através da produção e desenvolvimento das atividades programadas. Por fim, era implementado um questionário referente ao tema trabalhado, a fim de coletar dados sobre a temática, bem como avaliar e conhecer a opinião do grupo sobre a atividade proposta. CONCLUSÃO: Evidenciou-se a carência de informação e atenção que os participantes possuíam. Sendo assim, o projeto de extensão pode promover uma conscientização aos envolvidos de forma dinâmica e criativa, alcançando os objetivos propostos.
\end{abstract}

Descritores: Extensão universitária. Saúde do Idoso. Enfermagem. Educação e saúde. Envelhecimento.

\begin{abstract}
OBJECTIVE: To report the actions, through an experience report, of students aimed to promoting healthy aging in the municipality of Cuité - PB. METHODOLOGY: It reports experiences of undergraduate students in the Bachelor of Nursing on performance of its extension activities in the project entitled "Meeting of generations in promotion of healthy aging", during the period of May and December 2013 at the Federal University of Campina Grande, PB, Cuité campus. RESULTS AND DISCUSSION: The project was developed based on six steps: work team training; approximation to the target audience; information gathering of the target audience; production and manufacture of the material; workshops about health education and scientific papers. During the term of the project were realized sixteen workshops and eight different themes were performed. The workshops were held with the main focus on the stimulation of the elderly participation. The subjects were explained succinctly and dynamically, by students, in order to hold the attention of participants. Then the elderly interacted actively through the production and development of scheduled activities. Finally it was implemented a questionnaire related to the worked subject in order to collect data, as well as assess and meet the group's opinion on the proposed activity. CONCLUSION: The reports evidenced the lack of information and attention that the participants had. Thus, an extension project can promote the awareness of the involved in a dynamically and creatively way, reaching those goals.
\end{abstract}

Keywords: University Extension. Health of the Elderly. Nursing. Education and health. Aging. 


\section{INTRODUÇÃO}

As universidades cada vez mais buscam formar profissionais conscientes de suas responsabilidades sociais, a fim de superar as desigualdades existentes, para tanto é necessário que os acadêmicos tenham uma efetiva interação com a sociedade que é viabilizada por meio da extensão universitária. A extensão é um método educativo, cultural e científico que associa o ensino e a pesquisa, com as necessidades dos diversos setores da sociedade, possibilitando a formação de um espaço privilegiado de troca de conhecimentos (Brasil, 2008).

A prática da extensão na Universidade Federal de Campina Grande (UFCG) foi regulamentada pela resolução $\mathrm{N}^{\mathrm{o}} 02$ de 07 de dezembro de 2004, da Câmara Superior de Pesquisa e Extensão - CSPE. A extensão nesta instituição de ensido é realizada sob a forma de atendimento direto à comunidade pelos órgãos de administração, ou de ensino e pesquisa, através de eventos, programas e projetos de extensão.

Os programas de extensão da UFCG consciste em um conjunto de ações extensionistas inter-relacionadas, com atividades interdisciplinares que podem ser de caráter permanente ou eventual, com objetivos gerais comuns e objetivos específicos distintos. Ademais, os projetos de extensão são conjuntos de ações processuais contínuas de caráter educativo, social, cultural, científico e tecnológico (UNIVERSIDADE FEDERAL DE CAMPINA GRANDE, Resolução $\mathrm{N}^{\circ}$ 02/2004).

A prática educativa é um dos principais eixos norteadores para a formação do profissional de Enfermagem, que se consolida nos múltiplos espaços de realização das suas ações, sejam elas desenvolvidas na comunidade ou nos serviços de saúde. Algumas medidas são capazes de potencializar essa prática, como por exemplo, a identificação de pessoas vulneráveis ao adoecimento e possíveis ambientes pedagógicos (ACIOLI, 2008).

Nesse contexto, este estudo traz uma atenção especial ao público idoso, tendo em vista a suas necessidades especiais e sua abrangência na sociedade atual. Deste modo, é relevante ressaltar que o processo de envelhecimento populacional é um fenômeno mundial e no Brasil, o seu desenvolvimento ocorre de forma acelerada. Estima-se que em 2020, o país será o sexto do mundo em número de idosos, apresentando um número superior a 30 milhões de pessoas idosas (VERAS, 2009).

Tendo em vista o ritmo acelerado do envelhecimento, envelhecer com qualidade de vida e bem-estar se torna um desafio e um objetivo almejado para a população 
(SILVEIRA; FARO, 2008). Para tanto é necessário entender esse estrato populacional a partir de um enfoque holístico da saúde, abarcando alimentação, atividade física, atividade mental e equilíbrio emocional, unificados no curso de sua vida (ALENCAR; BARROS JÚNIOR; CARVALHO, 2008).

Nessa pespectiva, foi criado em 2013 na UFCG, no Centro de Educação em Saúde, o projeto intitulado "Encontro de gerações na promoção do envelhecimento saudável”, o mesmo tinha o intuito de realizar atividades educativas, a fim de estimular a adoção de hábitos saudáveis pelos idosos do município de Cuité - Paraíba (PB). Revela-se que o projeto em questão atuou como um viés de prevenção primária e secundária nas problemáticas de saúde, através de práticas de educação em saúde.

A fim de colaborar para a reflexão e o fortalecimento das atividades de extensão no cenário acadêmico e na práxis da Enfermagem, viu-se a necessidade da realização deste estudo, através do relato das atividades realizadas por 10 (dez) extensionistas, graduandos do curso Bacharelado em Enfermagem no desempenho de suas atividades de extensão, no período compreendido entre os meses de maio a dezembro de 2013. Assim, o presente ensaio tem como objetivo relatar as ações dos extensionistas voltadas para a promoção do envelhecimento saudável no município de
Cuité - PB através de um relato de experiência.

\section{METODOLOGIA}

Trata-se de um relato de experiência, acerca das vivências dos alunos extensionistas do projeto "Encontro de gerações na promoção do envelhecimento saudável" da UFCG, campus de Cuité, no período referente aos semestres 2013.1 e 2013.2, que corresponde aos meses de maio a dezembro de 2013.

Neste sentido, este estudo é de natureza descritiva qualitativa. Segundo Lakatos e Marconi (2007), o método qualitativo analisa e interpreta aspectos mais profundos, descrevendo a complexidade e as peculiaridades do comportamento humano, permitindo uma análise mais delineada sobre as investigações, hábitos, atitudes e tendências do comportamento. De acordo com Cervo; Berviam; Da Silva (2007) a descrição observa, registra e analisa as características de uma população ou fenômeno, sem manipulá-las, por meio de técnicas padronizadas de coleta de dados, tais como a observação sistemática. Para tanto, realizou-se uma análise crítica das atividades desenvolvidas durante o período subscrito e dos relatórios desenvolvidos ao final de cada ação realizada. 
No âmbito do projeto, as atividades foram desenvolvidas por alunos que já havia cursardo a disciplina de enfermagem na saúde do idoso. Ademais, os estudantes foram aprovados em processo seletivo instituído no primeiro semestre de 2013, por intermédio da solicitação da Coodenadora do projeto, da diretoria acadêmica da UFCG em concordância com a coordenação do curso de enfermagem e da Pró-Reitoria de Pesquisa e Extensão. O mesmo constituiu-se de uma dissertação sobre a saúde do idoso, entrevista com a Coodenadora do projeto e análise do rendimento acadêmico dos candidatos. Nesse processo, foram selecionados 10 (dez) extensionistas, sendo 1 (um) bolsista e 9 (nove) volntários, os quais realizaram suas atividades em uma Unidade Básica de Saúde da Família e no Centro de Assistência Social do município de Cuité-PB.

Do ponto de vista prático o relato de experiência vai além de uma simples descrição sucinta sobre uma atividade, pois por meio dele, é possível conhecer com maior domínio a experiência descrita. Permite ainda, compará-lo com outros ensaios semelhantes, possibilitando uma maior compreensão sobre o tema abordado. Portanto, a realização deste estudo é justificável, pois permitirá uma maior discussão sobre a temática trabalhada, além de prover elementos para o desenvolvimento de novos estudos.

\section{RESULTADOS E DISCUSSÃO}

Segundo o Ministério da Saúde, o envelhecimento populacional resulta de alterações referentes a indicadores de saúde, como a diminuição da mortalidade e da fecundidade, associada a avanços tecnológicos no que se refere a saúde e bem estar, acarretando assim, no aumento da expectativa de vida (BRASIL, 2006).

Conforme Censo realizado pelo Instituto Brasileiro de Geografia e Estatística (IBGE) no ano de 2010 o município de Cuité no estado da Paraíba (Brasil), localizado na microrregião do Curimataú Ocidental Paraibano, tem uma população de 19.978 habitantes, e destes 3.041 habitantes estão entre a faixa etária de 60 anos ou mais, do sexo feminino e masculino.

Neste sentido, considerando que a população do município de Cuité abrange um quantitativo significativo na faixa etária igual ou superior aos 60 anos, percebemos a importância da realização de ações que contribuíssem para a melhoria da qualidade de vida destes idosos. Deste modo, viu-se a necessidade da implementação de um Projeto de Extensão no município que contemplasse este público alvo.

$$
\text { É notório que os programas de }
$$
promoção à saúde dos idosos são cada vez mais necessários. Conforme Araújo et al. (2011) atualmente, a maioria destes 
programas são realizados no âmbito público ou da extensão universitária. Na sua maioria, realizam atividades que visam o envelhecimento ativo e saudável, buscando-se a longevidade, a manutenção da capacidade funcional e preservação da autonomia do idoso.

No projeto de extensão "Encontro de gerações na promoção do envelhecimento saudável", o objetivo geral foi à implementação de práticas educativas e de cuidados voltadas para a população idosa que é atendida nas Unidades Básicas de Saúde da Família (UBSF) do município de Cuité-PB, a fim de promover a saúde e prevenir agravos. $\mathrm{E}$ os objetivos específicos: Incentivar o emprego de ações de cuidado ao idoso, por meio de atividades dinâmicas e lúdicas; e promover oficinas de educação em saúde para a população idosa, voltadas para a promoção da saúde, prevenção e controle das doenças.

O público alvo do projeto foi à população idosa cadastrada nas Unidades Básicas de Saúde da Família (UBSF) da Zona Urbana do município de Cuité. Os encontros foram realizados semanalmente em uma UBSF e no Centro de Assistência Social do município referido anteriormente, totalizando 80 (oitenta) idosos, entre homens e mulheres.

Buscando-se almejar os objetivos propostos, a metodologia utilizada no decorrer da vigência constou de seis etapas: capacitação da equipe de trabalho;
Aproximação com o público alvo; Resgate das informações do público alvo; Produção e confecção do material; A educação em saúde para um cuidado adequado (realização de oficinas) e Produção de trabalhos científicos.

Ao decorrer do Projeto foram realizadas 16 oficinas e 8 temáticas diferentes foram trabalhadas. A oficina intitulada “Acolhimento nos serviços de Saúde", teve como objetivo realizar um primeiro contato e estabelecimento de vínculos com os idosos. A segunda, "Exercitando a Memória" na qual se buscou conhecer o grau de cognição dos idosos e estimular os mesmos a trabalharem a memória. A terceira, a quarta e a quinta com os temas respectivos: "Alimentação saudável: um compromisso com a saúde", com o intuito de incentivá-los a adoção de hábitos alimentares mais saudáveis e conforme a realidade de cada um. "Sorriso Grisalho: promovendo a saúde bucal", nesta ação objetivou-se orientá-los quanto a importância da higienização correta da cavidade oral e das próteses. Na oficina "Viva bem com o seu corpo: pratique atividade física", buscou instruí-los acerca da relevância da prática regular de atividades físicas para a prevenção de doenças, adoção de um estilo de vida mais saudável e também incentivar os mesmos a praticarem atividade física regularmente. Foram realizadas ainda as oficinas: "Prevenção de Quedas em Idosos", nesta foi ensinados meios de como deixar os ambientes 
da casa mais seguros, bem como os fatores que propiciam quedas, a fim de evitar e prevenir as quedas em idosos no ambiente domiciliar. Na oficina intitulada "Uso correto de medicamentos pelos idosos", os objetivos propostos foram orientar quanto ao uso correto de medicamentos e sanar possíveis dúvidas. Outra oficina realizada foi: "O uso de plantas medicinais entre idosos", na mesma almejou-se desmitificar as práticas errôneas do preparo e utilização das plantas medicinais e conhecer as terapias não farmacológicas utilizadas pelo grupo.

As oficinas foram realizadas com foco principal a estimulação participativa do idoso. Onde os temas eram explanados de forma sucinta e dinâmica, pelos extensionistas, a fim de prender a atenção dos participantes. Em seguida, os idosos interagiam de forma ativa através da produção e desenvolvimento das atividades programadas pela equipe de alunos anteriormente. Por fim, os extensionistas implementavam um questionário referente ao tema trabalhado durante a oficina, a fim de coletar dados sobre a temática, bem como avaliar e conhecer a opinião do grupo sobre a atividade proposta.

O projeto abarcou temas de relevância fundamental ao envelhecimento saudável, surgindo como um elo entre a informação e os idosos, por meio da educação em saúde. Este projeto trouxe para os idosos, ações educativas de conscientização, em especial para a promoção de um envelhecimento saudável, prevenindo agravos através da mudança no estilo de vida. Percebemos sua importância uma vez que a extensão universitária promove benefícios para os extensionistas bem como para a comunidade, por intermédio do aprendizado e troca mútua de conhecimentos.

O contato entre comunidade e extensionistas proporciona o crescimento de ambos, pois através da realização de oficinas voltadas para a comunidade os discentes tem a oportunidade de colocar em prática os conhecimentos adquiridos durante a graduação, conhecer a realidade a qual a população está inserida e ao mesmo tempo em que ocorre uma troca de experiência e conhecimento entre os envolvidos (SILVA, 2009).

Assim, o desenvolvimento das ações extensionistas no município de Cuité do projeto de intitulado "Encontro de Gerações na Promoção do Envelhecimento Saudável" contribuíu para aumentar o vínculo universidade/comunidade, priorizando ações voltadas para a Saúde do Idoso.

\section{CONSIDERAÇÕES FINAIS}

Percebe- se que o aumento na esperança de vida consiste numa conquista da humanidade. Contudo, a elevação do número 
de pessoas idosas traz consigo os problemas inerentes do envelhecimento, necessitando de uma visão ampla acerca do assunto e promoção de políticas e ações voltadas ao idoso que promovam sua qualidade de vida.

As práticas educativas para os idosos no projeto permitiram um maior conhecimento sobre os agravos à saúde e os seus meios de prevenção, favorecendo um entendimento prático das situações. Nesse interim, ressalta-se a responsabilidade dos extensionistas, que eram acadêmicos de enfermagem, enquanto futuros profissionais de saúde, em proporcionar as condições adequadas ao processo de educação em saúde, para favorecer a mudança nos hábitos de vida dos idosos participantes.

Evidenciou-se a carência de informação e atenção que os participantes possuíam. Sendo assim, o projeto de extensão

\section{REFERÊNCIAS}

ALENCAR, M.S.S.; BARROS JÚNIOR, F. O.; CARVALHO, C. M. R. G. Os aportes sóciopolíticos da educação nutricional na perspectiva de um envelhecimento saudável. Rev. Nutr.,

Campinas, v. 21, n.4, p. 369-381, jul/ago 2008.

Disponível em <

http://www.scielo.br/scielo.php?pid=S1415-

$52732008000400001 \&$ script=sci_arttext $>$ Acesso em 10 de setembro de 2014. pode promover uma conscientização aos envolvidos de forma dinâmica e criativa, alcançando os objetivos propostos.

Fica evidente o papel de contribuição social que a Extensão Universitária possui. A Universidade necessita apresentar a concepção do que a extensão apresenta para a sociedade em geral. Consiste em colocar em prática, pelos estudantes, aquilo que foi aprendido em sala de aula e implementar em ambiente extra universitário. Nesse contato entre estudantes e sociedade beneficiada, acontece o benefício mútuo, caracterizando a extensão universitária.

Conclui-se nesse estudo a importância da extensão universitária para a formação de profissionais com uma visão crítica, como também para benefício da sociedade, sendo a Atenção Básica um cenário propício para construção de ações educativas de saúde.

ACIOLI, S. A prática educativa como expressão do cuidado em Saúde Pública. Rev. bras.

enferm., Brasília, v. 61, n. 1, fev 2008.

Disponível em: <

http://www.scielo.br/scielo.php?pid=S0034$71672008000100019 \&$ script $=$ sci_arttext $>$ Acesso em 10 de setembro de 2014.

ARAÚJO, L. F. et al. Evidências das contribuições dos programas de assistência ao idoso na promoção do envelhecimento saudável no Brasil. Rev Panan Salud Publica, v.30, n.1, 2011. Disponível em: < 
http://www.scielosp.org/scielo.php?pid=S102049892011000700012\&script=sci_arttext $>$ Acesso em 10 de setembro de 2014.

BRASIL. Ministério da Saúde.

\section{Envelhecimento e saúde da pessoa idosa.}

Secretaria de Atenção à Saúde. Departamento de Atenção Básica. Brasília: 2006

BRASIL. Ministério de Educação, Secretária de Ensino Superior. Plano Nacional de Extensão. Portal MEC - Sitio SESu. 2008. Disponível em: $<$ http://www.uems.br/portal/editais/pdi/planonacional-extensao.pdf.> Acesso em 24 de agosto de 2014.

CERVO, A. L.; BERVIAN, P. A.; SILVA, R. da. Metodologia Científica. 6. ed. São Paulo: Prentice Hall, 2007.

\section{INSTITUTO BRASILEIRO DE}

\section{GEOGRAFIA E ESTATÍSTICA. IBGE:}

Censo Demográfico 2010. Disponível em:

$<$ http://cidades.ibge.gov.br/painel/painel.php? lang $=\&$ codmun $=250510 \&$ search $=$ paraiba $\mid$ cuit e|infogr\%E1ficos:-dados-gerais-domunic\%EDpio>. Acesso em 10 de setembro de 2015.

LAKATOS, E. M.; MARCONI, M. de A. Fundamentos de metodologia científica. 6a.ed. São Paulo. Atlas, 2007.
SILVEIRA, S. C.; FARO, A. C. M. Contribuição da reabilitação na saúde e na qualidade de vida do idoso no Brasil: reflexões para a assistência multidisciplinar. Estud. interdiscip. envelhec., Porto Alegre, v. 13, n. 1, p. 55-62, 2008.

Disponível em: < http://www.seer.ufrgs.br/RevEnvelhecer/articl e/view/6947> Acesso em 11 de setembro de 2014.

UNIVERSIDADE FEDERAL DE CAMPINA GRANDE. RESOLUÇÃO CSPE/UFCG No 02 de 07 de dezembro de 2004. Disponível em: $<$ http://www.ufcg.edu.br/ costa/resolucoes/re s_15022004.pdf>. Acesso em 22 de Set. 2014.

VERAS, R. Envelhecimento populacional contemporâneo: demandas, desafios e inovações. Rev. Saúde Pública, vol.43, n.3, p. 548-554, maio/jun 2009. Disponível em: < http://www.scielo.br/scielo.php?pid=S0034$89102009005000025 \&$ script=sci_arttext $>$ Acesso em 6 de setembro de 2014. 